\title{
Genomic insights into the serine protease gene family and expression profile analysis in the planthopper, Nilaparvata lugens
}

\author{
Yan-Yuan Bao, Xia Qin, Bing Yu, Li-Bo Chen, Zhe-Chao Wang and Chuan-Xi Zhang*
}

\begin{abstract}
Background: The brown planthopper (Nilaparvata lugens) is one of the most destructive rice plant pests in Asia. $N$. lugens causes extensive damage to rice by sucking rice phloem sap, which results in hopper burn (complete death of the rice plants). Despite its importance, little is known about the digestion, development and defense mechanisms of this hemimetabolous insect pest. In this study, we aim to identify the serine protease (SP) and serine protease homolog (SPH) genes, which form a large family in eukaryotes, due to the potential for multiple physiological roles. Having a fully sequenced genome for $\mathrm{N}$. lugens allows us to perform in-depth analysis of the gene structures, reveal the evolutionary relationships and predict the physiological functions of SP genes.

Results: The genome- and transcriptome-wide analysis identified 90 putative SP (65) and SPH (25) genes in N. lugens. Detailed gene information regarding the exon-intron organization, size, distribution and transcription orientation in the genome revealed that many SP/SPH loci are closely situated on the same scaffold, indicating the frequent occurrence of gene duplications in this large gene family. The gene expression profiles revealed new findings with regard to how SPs/SPHs respond to bacterial infections as well as their tissue-, development- and sex-specific expressions.

Conclusions: Our findings provide comprehensive gene sequence resources and expression profiles of the N. lugens SP and SPH genes, which give insights into clarifying the potentially functional roles of these genes in the biological processes including development, digestion, reproduction and immunity.
\end{abstract}

Keywords: Nilaparvate lugens, Serine protease, Serine protease homolog, Genome, Transcriptome, Expression profile

\section{Background}

The brown planthopper Nilaparvata lugens Stål (Hemiptera: Delphacidae) is a typical phloem sap feeder and is considered to be one of the most devastating pests for rice throughout Asia. It sucks sap from rice phloem and transmits plant viruses, which can lead to a dramatic reduction in yield and cause significant economic loss [1]. The first line of defense in $N$. lugens management is the use of chemical insecticides; however, the overuse of insecticides has caused planthopper resurgence and environmental risks [2]. In recent years, the target gene silencing based on RNA interference (RNAi) technology has been considered for its feasibility and potential in protecting crops against agriculturally important lepidopteran and

\footnotetext{
* Correspondence: chxzhang@zju.edu.cn

State Key Laboratory of Rice Biology and Ministry of Agriculture Key Laboratory of Agricultural Entomology, Institute of Insect Sciences, Zhejiang University, Hangzhou 310058, China
}

coleopteran insect pests $[3,4]$. RNAi-mediated crop protection is promising because this strategy allows the suppression of gene expression in a wide range of potential targets [5]. The target genes may be useful for developing high efficiency and low toxicity insecticides [6]. Currently, there is an urgent need to develop RNAi-based technique to control highly destructive phloem-sucking hemipteran pests, such as planthoppers, aphids and whiteflies, for which no effective Bt (Bacillus thuringiensis) toxins exist.

Serine proteases (SPs) in the chymotrypsin (S1) family constitute one of the largest gene families of multifunctional enzymes that play important roles in various physiological processes, including digestion, development and the immune response [7]. They are the principal proteolytic digestive enzymes in certain insects and thus provide nutrients required for survival and fecundity. Almost all of the known members of the chymotrypsin family have been

\section{Biomed Central}

(c) 2014 Bao et al.; licensee BioMed Central Ltd. This is an Open Access article distributed under the terms of the Creative Commons Attribution License (http://creativecommons.org/licenses/by/4.0), which permits unrestricted use, distribution, and reproduction in any medium, provided the original work is properly credited. The Creative Commons Public Domain Dedication waiver (http://creativecommons.org/publicdomain/zero/1.0/) applies to the data made available in this article unless otherwise stated. 
found in animals. It is striking that no member of this very successful family has been encountered in protozoa, fungi or plants [8]. SPs are generally synthesized as zymogens, which require proteolysis at a specific site for activation. Enzymatically active SPs feature a high specificity catalytic triad in their catalytic domain, composed of histidine (His), aspartic acid (Asp) and serine (Ser). Biochemical and genomic analyses revealed that catalytically inactive serine protease homologs (SPH) are also members of the SP family [7]. SPHs have similar sequences to SPs but lack one or more of the catalytic residues. Nonproteolytic SPHs are important components of phenoloxidase activation in insect innate immune responses [9].

Genome-wide analyses of SP and SPH genes have been performed in Diptera Drosophila melanogaster [10], Hymenoptera Apis mellifera [7] and Lepidoptera Bombyx mori [11]. However, little is known about these genes in plant phloem sap-sucking Hemiptera insect species. Recently, we sequenced the whole $N$. lugens genome and obtained gene annotation information (Zhejiang University $N$. lugens genome project team). $N$. lugens genome is the first characterized genome of a monophagous sap-sucking arthropod herbivore. The $N$. lugens genomic information allows the global analysis of SP and $\mathrm{SPH}$ genes in this insect species. In our previous study, we performed transcriptome sequencing and gene expression analysis using the next-generation highthroughput Illumina technology, which provided the detailed gene expression information regarding the developmental stages, wing dimorphism, sex differences, immune responses and tissue specificity in $N$. lugens $[2,12,13]$. In this study, a thorough screening of the $N$. lugens genome sequence coupled with the available transcriptome datasets generated the comprehensive information of SP and SPH genes, which presents an overview of the gene structures, evolutionary relationships and the expression specificity of these genes. These data could be useful in identifying the potential target genes for insect pest management.

\section{Methods}

\section{Insects}

The $N$. lugens strain was originally obtained from local rice fields in the Huajiachi Campus of Zhejiang University, Hangzhou, China. The insects employed in this study were the offspring of a single female and were reared at $26 \pm 0.5^{\circ} \mathrm{C}$ with $50 \pm 5 \%$ humidity on fresh rice seedlings under a 16:8 hour light:dark photoperiod as previously described $[2,12,13]$.

\section{Immunization and collection of tissues}

$N$. lugens 5th instar nymphs were immunized with a microinjection of heat-killed Escherichia coli K12 or Bacillus subtilis (500 cells per nymph, respectively) using the FemtoJet Microinjection System (Eppendorf-Nethler-Hinz, Hamburg, Germany). Nymphs were collected at different time points $(6,12$ and 24 hours) after infection for the bacteria-induced expression experiment as previously described [13].

For studies of tissue-specific expression, the $N$. lugens were dissected under a Leica S8AP0 stereomicroscope (Leica Microsystems GmbH, Wetzlar, Germany). The fat body, midgut, salivary gland, male reproductive system, ovary and carcass (the remaining body) were isolated and quickly washed in cold diethylpyrocarbonate (DEPC)treated $\mathrm{NaCl} / \mathrm{Pi}$ solution $(137 \mathrm{mM} \mathrm{NaCl}, 2.68 \mathrm{mM} \mathrm{KCl}$, $8.1 \mathrm{mM} \mathrm{Na} \mathrm{HPO}_{4}, 1.47 \mathrm{mM} \mathrm{KH_{2 }} \mathrm{PO}_{4}, \mathrm{pH}$ 7.4) and immediately frozen at $-80^{\circ} \mathrm{C}$ as previously described $[2,13]$. For each tissue, more than $300 \mathrm{~N}$. lugens individuals were dissected and used for RNA extractions.

\section{Quantitative real-time PCR (qRT-PCR) analysis}

Total RNA was extracted from $N$. lugens nymphs or adults using RNAiso plus based on the manufacturer's protocol (TaKaRa, Dalian, China). As previously described [2,13], the RNA concentration was adjusted with RNase-free water to $1 \mu \mathrm{g} / \mu \mathrm{l}$, and $1 \mu \mathrm{g}$ of RNA was used for reverse transcription in a $10 \mu \mathrm{l}$ reaction using the ReverTra Ace ${ }^{\bullet}$ qPCR RT Master Mix with gDNA Removal Kit (ToYoBo, Osaka, Japan) to remove any contaminating genomic DNA. Quantitative RT-PCR was carried out on a CFX96 $6^{\text {Th }}$ Real-Time PCR Detection System (Bio-Rad, Hercules, CA, USA) using the $\mathrm{iQ}^{\text {tw }} \mathrm{SYBR}^{\circ}$ Green Supermix Kit (Bio-Rad). The firststrand cDNA and the no-template control (nucleasefree water) were used as templates for three biological replication assays under the following conditions: denaturation at $95^{\circ} \mathrm{C}$ for $2 \mathrm{~min}$, followed by 40 cycles at $95^{\circ} \mathrm{C}$ for $15 \mathrm{~s}$ and $60^{\circ} \mathrm{C}$ for $30 \mathrm{~s}$. Melting curves were constructed following amplifications and the data were analyzed using the Bio-Rad CFX Manager 2.1 software. The specific primers for amplifying the SP/SPH genes were designed based on the $N$. lugens transcriptomic sequences (accession number: SRX023419) that have been submitted to the Sequence Read Archive (SRA) database (http://www.ncbi.nlm.nih.gov/sra), as shown in an Additional file 1: Table S1). The expression of the $N$. lugens 18 s rRNA gene as an internal control (GenBank accession no. JN662398) was analyzed using the following primers: 5'-CGCTACTACCGATTGAA-3' (sense) and 5'-GGAAACCTTGTTACGACTT -3' (antisense). The use of reference genes as internal controls is the most appropriate normalization strategy for achieving the reliable qRT-PCR assay [14]. In our previous study, the utility of $N$. lugens $18 \mathrm{~s}$ rRNA gene has been validated for their stably expressions in $N$. lugens tissues, developmental stage and immune-induced individuals $[2,12,13]$. In this study, the results were normalized to the expression level 
of $N$. lugens $18 \mathrm{~s}$ rRNA. An NTC sample was run to detect any contamination and to determine the degree of dimer formation. The relative quantitative method ( $\Delta \Delta \mathrm{C}_{\mathrm{t}}$ method, $\mathrm{C}_{\mathrm{t}}$ is the threshold cycle) was used to evaluate the relative differences in the transcript levels [15]. Namely, the following equation was used: $\Delta C_{t}=$ the $C_{t}$ of SP gene - the $C_{t}$ of the $18 \mathrm{~s}$ rRNA gene.

\section{Identification of SP and SPH genes from the genome of $N$. lugens and differential expression analysis}

The SP and SPH genes were searched against the $N$. lugens genome sequence based on the KEGG (ftp://ftp. uniprot.org/pub/databases/uniprot/, v58), Swissprot (ftp://

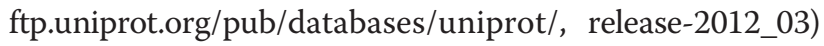
and Trembl (ftp://ftp.uniprot.org/pub/databases/uniprot/, release-2012_03) annotations. Predicted coding sequences of SP and SPH genes were used as reference sequences to match the $N$. lugens transcriptomic sequences using the tBLASTX algorithm with a cut-off E-value of $10^{-10}$. The deduced protein domains and signal peptides were determined by using Pfam (http://pfam.xfam.org/), SMART (http://smart.embl.de/) and InterProScan (http:// www.ebi.ac.uk/Tools/pfa/iprscan/) as previously described [13].

Owing to the accomplishment of the gene expression profiles of the differences between the $N$. lugens development and sex genes in our previous study [12], we are able to analyze the development- and sex-specific expressions of SP and SPH genes. We used a FDR (false discovery rate) $<0.001$ as a threshold to judge significant differences in gene expression.

\section{Comparison of trypsin-like genes in insect genomes}

Trypsin-like genes were identified in the available genomes of the following insect species: Apis mellifera (hymenopteragenome.org/drupal/sites/hymenopteragenome. org.beebase/files/data/); Aedes aegypti (http://www.vector base.org/GetData/Downloads/); Acyrthosiphon pisum (www. inra.fr/aphidbase/); Drosophila melanogaster (ftp.flybase.org/ genomes/Drosophila_melanogaster/dmel_r5.27_FB2010_04/);

Anopheles gambiae (ftp.vectorbase.org/public_data/ organism_data/aaegypti/Geneset/); Tribolium castaneum (ftp://ftp.ncbi.nih.gov/genomes/Tribolium_castaneum); Culex quinquefasciatus (http://www.vector base.org/GetData/Downloads/); and Bombyx mori (ftp:// silkdb.org/pub/release_2.0/), based on the KEGG (ftp://ftp. uniprot.org/pub/databases/uniprot/) and Swissprot (ftp:// ftp.uniprot.org/pub/databases/uniprot/) annotations as previously described [13].

\section{Phylogenetic analysis}

The functional serine protease domains of the N. lugens SPs and SPHs were aligned with the best-matched homologs of other insect species using the ClustalX program [16]. The phylogenetic trees were constructed by the maximum likelihood (ML) method using the program Mega 5.05 (http://www.megasoftware.net/) [17]. Homologous relationships were determined using bootstrap analysis with 1000 replications.

\section{Results and discussion \\ Identification of SP and SPH genes in the $N$. lugens genome}

We identified a total of 90 predicted serine protease-like genes by searching the $N$. lugens genome sequence based on the KEGG, Swissprot and Trembl annotations, which were validated using the tBLASTX algorithm with a cut-off E-value of $10^{-10}$ (Table 1). Most of these genes belong to the chymotrypsin (S1) family. Based on the presence or absence of the catalytic triad essential for the catalytic activity, we classified these serine proteaselike genes as SPs and SPHs, respectively. Sixty-five genes (SP) possess the intact catalytic triads, while twenty-five genes (SPH) lack one or more active site residues, suggesting that they could have lost catalytic function. Most of SPs and SPHs contain putative signal peptides and are expected to be extracellular proteases; only four SPs display transmembrane regions in their sequences. The predicted sequences reveal that approximately $70 \%$ of the SPs and SPHs are similarly sized (approximately 300 amino acid residues), while a few of them are larger (more than 500 amino acid residues) and contain specific modules, such as Clip domain, complement control protein $(\mathrm{CCP})$ domain, low-density lipoprotein receptor class A (LDLA) domain, myb/SANT-like (MADF) domain, complement CUB domain, frizzled (FRI) domain and scavenger receptor Cys-rich (SR) domain. Many SP and $\mathrm{SPH}$ genes display a tandem repeat distribution at the same scaffolds, implicating that gene duplication frequently occurred in this gene family. We roughly classified the $N$. lugens serine protease-like genes into three major clades according to their potential functions.

\section{Trypsin-like genes}

A total of 31 trypsin-like genes were identified from the $N$. lugens genome (Table 1). Trypsins usually have a definite structure, which includes a signal peptide and a catalytic serine protease domain. Of the 31 trypsin-like genes, 27 contain a putative signal peptide sequence, suggesting that they are secreting-type proteins. Based on the presence or absence of the catalytic triads, the $N$. lugens trypsin-like genes were clustered into 22 SPs and 9 SPHs, respectively. The catalytic triad, His, Asp and Ser residues, is highly conserved in the SP sequence motif TAAHC, DIAL and GDSGGP, indicating the active proteases (Figure 1). One or more residues of the catalytic triad are missing in the $N$. lugens SPHs, suggesting that these proteases could have lost their 
Table 1 The genomic prediction of $N$. lugens serine protease and serine protease homologs

\begin{tabular}{|c|c|c|c|c|c|c|c|c|c|c|c|}
\hline Predicted gene & GenBank ID & Locus & Size (aa) & Exon & Orientation & HDS & Domains/Motifs & Best match & Similarity & E-value & Cover \\
\hline \multicolumn{12}{|c|}{ Trypsin-like serine protease } \\
\hline Trypsin 1 & KJ512112 & scaffold574 & 334 & 7 & + & active & Signal P & P. h.corporis & $47 \%$ & $1 e-28$ & $95 \%$ \\
\hline Trypsin 2 & KJ512113 & scaffold261 & 360 & 7 & - & active & Signal P & P. h.corporis & $79 \%$ & $5 e-137$ & $78 \%$ \\
\hline Trypsin 3 & KJ512114 & scaffold998 & 299 & 6 & - & active & & P. h.corporis & $62 \%$ & $4 e-91$ & $99 \%$ \\
\hline Trypsin 4 & KJ512115 & scaffold106 & 795 & 5 & - & active & Signal P & P. h.corporis & $84 \%$ & $4 e-178$ & $41 \%$ \\
\hline Trypsin 5 & KJ512116 & scaffold126 & 347 & 3 & - & active & Signal P & P. h.corporis & $69 \%$ & 1e-114 & $90 \%$ \\
\hline Trypsin 6 & KJ512117 & scaffold126 & 360 & 7 & + & active & Signal P & P. h.corporis & $79 \%$ & $5 e-137$ & $78 \%$ \\
\hline Trypsin 7 & KJ512118 & scaffold64 & 393 & 4 & - & active & Signal P & P. h.corporis & $89 \%$ & 0 & $84 \%$ \\
\hline Trypsin 8 & KJ512119 & scaffold260 & 323 & 5 & + & active & Signal P & P. h.corporis & $65 \%$ & $3 e-95$ & $91 \%$ \\
\hline Trypsin 9 & KJ512120 & scaffold189 & 318 & 12 & - & active & Signal P & N.lugens & $98 \%$ & 0 & $100 \%$ \\
\hline Trypsin 10 & KJ512121 & scaffold707 & 303 & 8 & - & active & Signal $P$ & N.lugens & $54 \%$ & $4 e-58$ & $79 \%$ \\
\hline Trypsin 11 & KJ512122 & scaffold1712 & 290 & 5 & + & active & Signal P & C.quinquefasciatus & $54 \%$ & $2 \mathrm{e}-46$ & $92 \%$ \\
\hline Trypsin 12 & KJ512123 & scaffold172 & 295 & 12 & - & active & Signal P & A.mellifera & $53 \%$ & $6 e-44$ & $90 \%$ \\
\hline Trypsin 13 & KJ512124 & scaffold6559 & 423 & 3 & - & active & Signal $P$ & D.pteronyssinus & $59 \%$ & $9 e-50$ & $77 \%$ \\
\hline Trypsin 14 & KJ512125 & scaffold6559 & 287 & 1 & - & active & Signal P & D.pteronyssinus & $58 \%$ & $1 e-53$ & $79 \%$ \\
\hline Trypsin 15 & KJ512126 & scaffold6559 & 731 & 7 & - & active & Signal P & D.pteronyssinus & $61 \%$ & $8 e-66$ & $99 \%$ \\
\hline Trypsin 16 & KJ512127 & scaffold6559 & 262 & 2 & - & active & Signal $P$ & C.capitata & $51 \%$ & $2 \mathrm{e}-27$ & $96 \%$ \\
\hline Trypsin 17 & KJ512128 & scaffold50 & 318 & 6 & + & active & Signal $P$ & C.felis & $59 \%$ & $4 e-64$ & $89 \%$ \\
\hline Trypsin 18 & KJ512129 & scaffold327 & 292 & 5 & - & active & Signal P & A.mellifera & $48 \%$ & $5 e-25$ & $79 \%$ \\
\hline Trypsin 19 & KJ512130 & scaffold126 & 511 & 7 & - & active & Signal $P$ & T.castaneum & $68 \%$ & 0 & $94 \%$ \\
\hline Trypsin 20 & KJ512131 & scaffold577 & 294 & 1 & + & active & Signal P & D.melanogaster & $48 \%$ & $5 e-32$ & $87 \%$ \\
\hline Trypsin 21 & KJ512132 & scaffold1720 & 272 & 4 & - & active & & H. saltator & $48 \%$ & $4 e-20$ & $70 \%$ \\
\hline Trypsin 22 & KJ512133 & scaffold1722 & 747 & 14 & + & active & & A.pisum & $54 \%$ & $2 e-52$ & $75 \%$ \\
\hline Trypsin 23 & KJ512134 & scaffold299 & 260 & 6 & - & inactive & Signal $P$ & N.vitripennis & $45 \%$ & $4 e-25$ & $85 \%$ \\
\hline Trypsin 24 & KJ512135 & scaffold299 & 290 & 5 & - & inactive & Signal P & N.lugens & $98 \%$ & 0 & $94 \%$ \\
\hline Trypsin 25 & KJ512136 & scaffold299 & 300 & 7 & - & Inactive & Signal P & N.lugens & $56 \%$ & $4 e-49$ & $82 \%$ \\
\hline Trypsin 26 & KJ512137 & scaffold299 & 292 & 5 & - & Inactive & Signal P & N.lugens & $50 \%$ & $4 e-53$ & $84 \%$ \\
\hline Trypsin 27 & KJ512138 & scaffold299 & 266 & 6 & - & inactive & Signal $P$ & N.vitripennis & $45 \%$ & $7 e-25$ & $97 \%$ \\
\hline Trypsin 28 & KJ512139 & scaffold601 & 298 & 1 & - & inactive & Signal P & T.castaneum & $48 \%$ & $3 e-23$ & $74 \%$ \\
\hline
\end{tabular}


Table 1 The genomic prediction of $\boldsymbol{N}$. lugens serine protease and serine protease homologs (Continued)

\begin{tabular}{|c|c|c|c|c|c|c|c|c|c|c|c|}
\hline Trypsin 29 & KJ512140 & scaffold682 & 325 & 6 & + & inactive & Signal $P$ & P. h.corporis & $66 \%$ & $3 e-106$ & $95 \%$ \\
\hline Trypsin 30 & KJ512141 & scaffold2065 & 281 & 6 & + & inactive & Signal P & D. yakuba & $55 \%$ & $6 e-34$ & $79 \%$ \\
\hline Trypsin 31 & KJ512142 & scaffold4540 & 247 & 4 & - & inactive & & P. h.corporis & $53 \%$ & $2 e-31$ & $91 \%$ \\
\hline \multicolumn{12}{|l|}{ Clotting serine proteases } \\
\hline Clotting factor $C$ like & KJ512060 & scaffold437 & 553 & 2 & + & active & CCP & C.biroi & $52 \%$ & $1 e-108$ & $96 \%$ \\
\hline Clotting factor B like & KJ512061 & scaffold867 & 321 & 7 & - & inactive & Signal P & B.impatiens & $53 \%$ & $1 e-52$ & $82 \%$ \\
\hline Proclotting enzyme 1 & KC355213 & scaffold424 & 397 & 7 & + & active & Clip & A.pisum & $56 \%$ & $4 e-91$ & $91 \%$ \\
\hline Proclotting enzyme 2 & KC355214 & scaffold424 & 376 & 12 & - & active & Clip & A.pisum & $55 \%$ & $6 e-89$ & $91 \%$ \\
\hline Proclotting enzyme 3 & KC355215 & scaffold1854 & 460 & 9 & - & active & Clip & C.floridanus & $66 \%$ & $4 e-73$ & $90 \%$ \\
\hline \multicolumn{12}{|l|}{ Serine protease nudel } \\
\hline Serine protease nudel like & KJ512077 & scaffold771 & 683 & 11 & - & active & LDLA & A.pisum & $47 \%$ & $2 e-86$ & $97 \%$ \\
\hline \multicolumn{12}{|l|}{ Serine protease gd } \\
\hline Serine protease gd like & KJ512078 & scaffold50 & 441 & 10 & + & active & Signal P & A.florea & $57 \%$ & $3 e-96$ & $94 \%$ \\
\hline \multicolumn{12}{|l|}{ Serine protease snake } \\
\hline Serine protease snake 1 & KC355219 & scaffold407 & 363 & 7 & + & active & Clip & A.pisum & $54 \%$ & $3 e-74$ & $89 \%$ \\
\hline Serine protease snake 2 & KC355220 & scaffold183 & 406 & 5 & - & active & Clip & A.pisum & $50 \%$ & $7 e-71$ & $99 \%$ \\
\hline Serine protease snake 3 & KC355221 & scaffold183 & 406 & 7 & - & active & Clip & A.pisum & $47 \%$ & $1 e-71$ & $99 \%$ \\
\hline Serine protease snake 4 & KC355222 & scaffold3538 & 546 & 7 & + & active & Clip & T.castaneum & $58 \%$ & $3 e-65$ & $95 \%$ \\
\hline Serine protease snake 5 & KC355223 & scaffold407 & 358 & 8 & - & inactive & Clip & A.pisum & $41 \%$ & $8 e-31$ & $89 \%$ \\
\hline Serine protease snake 6 & KC355224 & scaffold407 & 374 & 7 & - & inactive & Clip & A.pisum & $45 \%$ & $1 e-33$ & $93 \%$ \\
\hline Serine protease snake 7 & KC355225 & scaffold407 & 362 & 7 & - & active & Clip & A.pisum & $53 \%$ & $5 e-70$ & $85 \%$ \\
\hline Serine protease snake 8 & KJ512098 & scaffold407 & 389 & 7 & - & active & & A.pisum & $60 \%$ & $1 \mathrm{e}-74$ & $86 \%$ \\
\hline Serine protease snake 9 & KJ512099 & scaffold407 & 489 & 6 & + & active & Signal P & A.pisum & $57 \%$ & $4 \mathrm{e}-70$ & $56 \%$ \\
\hline Serine protease snake 10 & KJ512100 & scaffold407 & 367 & 7 & - & active & Signal P & A.pisum & $56 \%$ & $3 e-66$ & $80 \%$ \\
\hline Serine protease snake 11 & KJ512101 & scaffold4413 & 489 & 7 & - & active & Signal P & A.pisum & $60 \%$ & $2 e-69$ & $56 \%$ \\
\hline Serine protease snake 12 & KJ512102 & scaffold4413 & 389 & 6 & + & active & & A.pisum & $55 \%$ & $1 e-74$ & $86 \%$ \\
\hline \multicolumn{12}{|l|}{ Serine protease easter } \\
\hline Serine protease easter 1 & KJ512062 & scaffold258 & 289 & 3 & + & active & & H. saltator & $45 \%$ & $3 e-31$ & $97 \%$ \\
\hline Serine protease easter 2 & KJ512063 & scaffold258 & 319 & 6 & + & active & Signal P & H. saltator & $52 \%$ & $2 e-45$ & $98 \%$ \\
\hline Serine protease easter 3 & KJ512064 & scaffold258 & 397 & 7 & + & active & & N.vitripennis & $51 \%$ & $2 \mathrm{e}-27$ & $61 \%$ \\
\hline
\end{tabular}


Table 1 The genomic prediction of $\boldsymbol{N}$. lugens serine protease and serine protease homologs (Continued)

\begin{tabular}{|c|c|c|c|c|c|c|c|c|c|c|c|}
\hline Serine protease easter 4 & KJ512065 & scaffold258 & 407 & 7 & - & active & Signal P & H. saltator & $49 \%$ & $1 e-41$ & $76 \%$ \\
\hline Serine protease easter 5 & KJ512066 & scaffold258 & 334 & 5 & - & inactive & Signal P & H. saltator & $45 \%$ & $5 e-19$ & $80 \%$ \\
\hline Serine protease easter 6 & KJ512067 & scaffold258 & 330 & 6 & - & inactive & Signal $\mathrm{P}$ & H. saltator & $45 \%$ & $4 e-30$ & $92 \%$ \\
\hline Serine protease easter 7 & KJ512068 & scaffold574 & 315 & 7 & + & active & Signal P & H. saltator & $52 \%$ & $8 e-50$ & $92 \%$ \\
\hline Serine protease easter 8 & KJ512069 & scaffold574 & 323 & 5 & - & active & Signal P & H. saltator & $48 \%$ & $4 e-35$ & $98 \%$ \\
\hline Serine protease easter 9 & KJ512070 & scaffold1012 & 318 & 6 & + & inactive & Signal $\mathrm{P}$ & A.aegypti & $43 \%$ & $9 e-17$ & $80 \%$ \\
\hline Serine protease easter 10 & KJ512071 & scaffold1012 & 356 & 6 & + & inactive & Signal $P$ & A.aegypti & $43 \%$ & $2 e-17$ & $90 \%$ \\
\hline Serine protease easter 11 & KJ512072 & scaffold1012 & 322 & 7 & + & active & Signal $P$ & H. saltator & $50 \%$ & $5 e-41$ & $92 \%$ \\
\hline Serine protease easter 12 & KJ512073 & scaffold1121 & 334 & 6 & - & active & Signal P & H. saltator & $62 \%$ & $4 e-72$ & $85 \%$ \\
\hline Serine protease easter 13 & KJ512074 & scaffold236 & 310 & 10 & - & active & Signal $P$ & H. saltator & $52 \%$ & $2 e-43$ & $94 \%$ \\
\hline Serine protease easter 14 & KJ512075 & scaffold4778 & 307 & 8 & - & active & Signal $\mathrm{P}$ & H. saltator & $48 \%$ & $2 \mathrm{e}-32$ & $96 \%$ \\
\hline Serine protease easter 15 & KJ512076 & scaffold4872 & 340 & 4 & + & inactive & Signal P & A.mellifera & $45 \%$ & $5 e-20$ & $91 \%$ \\
\hline \multicolumn{12}{|l|}{ Serine protease stubble } \\
\hline Serine protease stubble like 1 & KJ512103 & scaffold126 & 324 & 7 & - & active & Signal $\mathrm{P}$ & M. rotundata & $78 \%$ & $9 e-148$ & $99 \%$ \\
\hline Serine protease stubble like 2 & KJ512104 & scaffold126 & 371 & 7 & + & active & Signal P & A.echinatior & $55 \%$ & $3 e-32$ & $70 \%$ \\
\hline Serine protease stubble like 3 & KJ512105 & scaffold223 & 398 & 7 & + & active & Signal P & B. mori & $64 \%$ & $8 e-119$ & $86 \%$ \\
\hline Serine protease stubble like 4 & KJ512106 & scaffold115 & 368 & 7 & - & active & & H. saltator & $79 \%$ & 0 & $100 \%$ \\
\hline Serine protease stubble like 5 & KJ512107 & scaffold886 & 467 & 5 & + & inactive & & $M$ rotundata & $62 \%$ & $4 e-129$ & $84 \%$ \\
\hline \multicolumn{12}{|l|}{ Serine protease } \\
\hline Serine protease 1 & KJ512079 & scaffold63 & 849 & 4 & + & inactive & & H. saltator & $71 \%$ & $2 \mathrm{e}-28$ & $26 \%$ \\
\hline Serine protease 2 & KJ512080 & scaffold1489 & 324 & 3 & - & active & Signal $P$ & B.mori & $52 \%$ & $4 e-31$ & $85 \%$ \\
\hline Serine protease 3 & KJ512081 & scaffold219 & 743 & 11 & + & active & MADF & A. pisum & $93 \%$ & $1 e-141$ & $64 \%$ \\
\hline Serine protease 4 & KJ512082 & scaffold1220 & 550 & 4 & + & active & CUB & A. pisum & $57 \%$ & $5 e-137$ & $88 \%$ \\
\hline Serine protease 5 & KJ512083 & scaffold1208 & 492 & 10 & + & active & CUB & A.pisum & $74 \%$ & $3 e-93$ & $83 \%$ \\
\hline Serine protease 6 & KJ512084 & scaffold1877 & 258 & 4 & + & inactive & Signal P & C.formosanus & $47 \%$ & $2 \mathrm{e}-20$ & $75 \%$ \\
\hline Serine protease 7 & KJ512085 & scaffold185 & 332 & 7 & + & active & Signal $\mathrm{P}$ & D.mojavensis & $55 \%$ & $1 \mathrm{e}-49$ & $74 \%$ \\
\hline Serine protease 8 & KJ512086 & scaffold762 & 346 & 6 & - & active & & T.castaneum & $65 \%$ & $2 e-83$ & $88 \%$ \\
\hline Serine protease HP21 & KJ512089 & scaffold2889 & 307 & 2 & - & inactive & Signal P & T.castaneum & $52 \%$ & $1 e-33$ & $86 \%$ \\
\hline Prophenoloxidase activating factor 1 & KJ512096 & scaffold66 & 390 & 7 & - & inactive & Signal $\mathrm{P}$ & T.molitor & $59 \%$ & $2 e-120$ & $100 \%$ \\
\hline Prophenoloxidase activating factor 2 & KJ512097 & scaffold66 & 395 & 6 & - & inactive & & T.molitor & $69 \%$ & $3 e-123$ & $83 \%$ \\
\hline
\end{tabular}


Table 1 The genomic prediction of $\boldsymbol{N}$. lugens serine protease and serine protease homologs (Continued)

\begin{tabular}{|c|c|c|c|c|c|c|c|c|c|c|c|}
\hline Hemolymph protease 1 & KJ512090 & scaffold972 & 314 & 1 & - & inactive & Signal P & T.castaneum & $47 \%$ & $1 e-32$ & $86 \%$ \\
\hline Hemolymph protease 2 & KJ512091 & scaffold236 & 314 & 6 & - & inactive & & C.quinquefasciatus & $43 \%$ & $7 e-16$ & $82 \%$ \\
\hline Serine protease SP24D & KJ512092 & scaffold2296 & 310 & 6 & + & active & Signal P & C.quinquefasciatus & $48 \%$ & $1 e-18$ & $78 \%$ \\
\hline Serine protease P69 & KJ512093 & scaffold598 & 708 & 8 & + & active & Signal P & T.castaneum & $69 \%$ & $7 e-84$ & $61 \%$ \\
\hline Serine protease HTRA2 & KJ512094 & scaffold552 & 420 & 7 & + & active & PDZ & N.vitripennis & $70 \%$ & $5 e-133$ & $91 \%$ \\
\hline \multicolumn{12}{|c|}{ Transmembrane serine protease } \\
\hline Serine protease 11 & KJ512108 & scaffold967 & 919 & 18 & - & active & FRI \& SR & T.castaneum & $72 \%$ & 0 & $86 \%$ \\
\hline Ovochymase 1 & KJ512109 & scaffold305 & 322 & 7 & + & active & TM & A.pisum & $77 \%$ & $3 e-124$ & $87 \%$ \\
\hline Ovochymase 2 & KJ512110 & scaffold 126 & 360 & 7 & - & active & TM & T.castaneum & $87 \%$ & $3 e-147$ & $68 \%$ \\
\hline Ovarian serine protease & KJ512111 & scaffold498 & 1334 & 9 & + & active & TM & T.castaneum & $71 \%$ & $2 \mathrm{e}-97$ & $47 \%$ \\
\hline
\end{tabular}

Table 1 Identification of SP and SPH genes in the $N$. lugens genome. The SP and SPH sequences were obtained from the $N$. lugens transcriptome databases and genomic sequences, which were confirmed using the tBLASTX algorithm with a cut-off E-value of $10^{-10}$. The genomic organization of exons and introns of the SP and SPH genes was predicted based on the mRNA-genome alignments at the NCBI spideyweb (http://www. ncbi.nlm.nih.gov/spidey/spideyweb.cgi). Locus, size and orientation indicate the location on scaffolds, predicted amino acids (aa) and the transcription orientation of the genes. HDS refers to the presence or absence of His, Asp and Ser residues in the catalytic triad, implying active or inactive proteases. A.pisum, Acyrthosiphon pisum; T. castaneum, Tribolium castaneum; P. h. corporis, Pediculus humanus corporis; B. mori, Bombyx mori; C.quinquefasciatus, Culex quinquefasciatus; C. capitata, Ceratitis capitata; H. saltator, Harpegnathos saltator; A. mellifera, Apis mellifera; M. rotundata, Megachile rotundata; A.echinatior, Acromyrmex echinatior; N. lugens, Nilaparvata lugens; C. felis, Ctenocephalides felis; D. pteronyssinus, Dermatophagoides pteronyssinus; D. melanogaster, Drosophila melanogaster; D.mojavensis, Drosophila mojavensis; D. yakuba, Drosophila yakuba C. floridanus, Camponotus floridanus; A. florea, Apis florea; N.vitripennis, Nasonia vitripennis; A. aegypti, Aedes aegypti; B. impatiens, Bombus impatiens; T. molitor, Tenebrio molitor; C. formosanus, Coptotermes formosanus; C. biroi, Cerapachys biroi. 


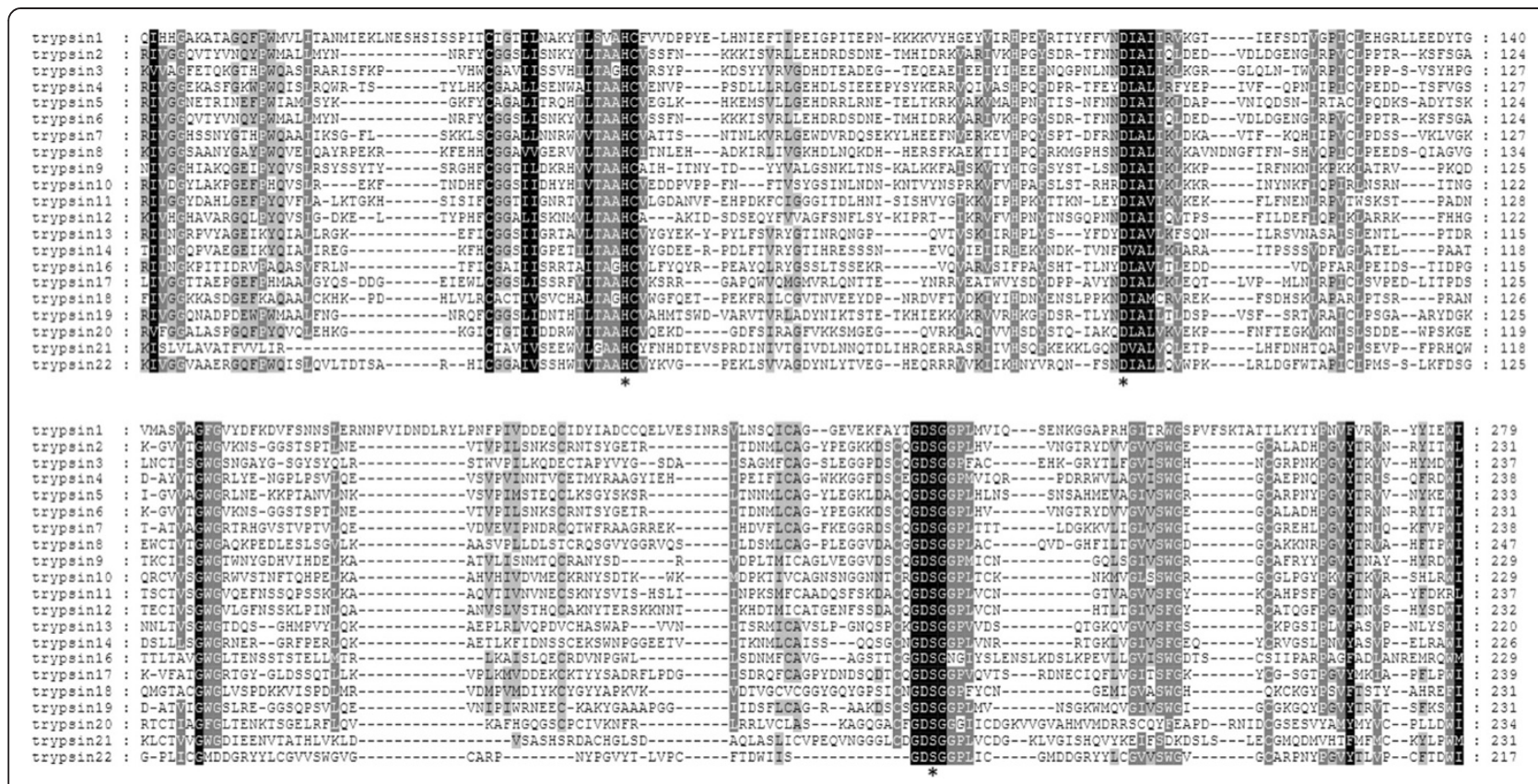

Figure 1 Multiple alignments of the serine protease domains of $\boldsymbol{N}$. lugens trypsins. The ClustalX program was used for the alignments. The conserved and the type-conserved residues in the deduced amino acid sequences are indicated in black and gray shades, respectively. The active triad consisting of histidine, aspartic acid and serine residues required for catalytic activity is marked by asterisks.

catalytic abilities. Most of the $N$. lugens trypsin-like genes showed significant sequence similarities with homologs of the hemipteran, phthirapteran and hymenopteran insect species, specifically, Acyrthosiphon pisum, Pediculus humanus corporis, Apis mellifera and Nasonia vitripennis (Table 1). However, three trypsinlike genes, trypsin 13, 14 and 15, did not show similarities with insect species, but displayed the highest sequence identity with Dermatophagoides pteronyssinus, a dust mite of non-insect arthropods.

Analysis of the gene structure revealed that most of trypsin-like genes consist of multiple exons (Table 1). Some trypsin-like genes forming gene clusters locate at the same scaffolds, i.e., the trypsin 5 and trypsin 6 genes locate at scaffold 126 with different transcription orientations. They contain three and seven exons flanked by the $5^{\prime}$ and $3^{\prime}$ untranslated regions (UTR5 and UTR3), respectively (Figure 2 and Table 1). The trypsin 13-16 genes closely locate at scaffold 6559 and the trypsin 23-27 genes locate at scaffold 299, which include 1-7 exons and have the same transcription orientations (Figure 2 and Table 1). The fact that two or more trypsin loci are located at the same scaffold implies that $N$. lugens might have undergone gene duplications in the genome. The trypsin 23-27 genes contain a signal peptide sequence but lack the complete catalytic triads in their serine protease domains, suggesting the possible absence of protease activity, while the trypsin 5-6 and 13-16 genes include both the signal peptide and catalytic triad, indicating that they may be the active proteases.

Trypsins were thought to be the digestive serine proteases. However, the gene expression information suggested that they may play multiple roles in $N$. lugens physiological process. In this study, we are interested in understanding their potential functions. Tissue specificity analysis showed the various expression patterns of the $N$. lugens trypsin-like genes. Trypsin 9-12, trypsin 23-27 and trypsin 30 were exclusively expressed in the midgut (Figure 3), which could be consistent with their potential function in digestive proteolysis. Interestingly, several trypsins displayed male or female-specific expression patterns, i.e., trypsin 18 was specifically expressed in ovary, while trypsin 1, 20-21, 28 and 31 were exclusively expressed in the male reproductive system, suggesting that they probably lack digestive functions and play important roles in the reproduction process of $N$. lugens instead. In contrast, trypsin 2 and 5 genes showed the high levels of tissue expression in the salivary gland, midgut, fat body and carcass, but almost no or extremely low levels in the male reproductive system and ovary, implying that these proteases possess multiply functions but not reproductive functions in the male and female individuals. Trypsin 8 and 17 seemed to have more extensive physiological roles including the potential female reproductive function as high transcript levels were detected in the ovary. Among the various tissues tested, trypsin 3 exhibited the highest transcript levels in 


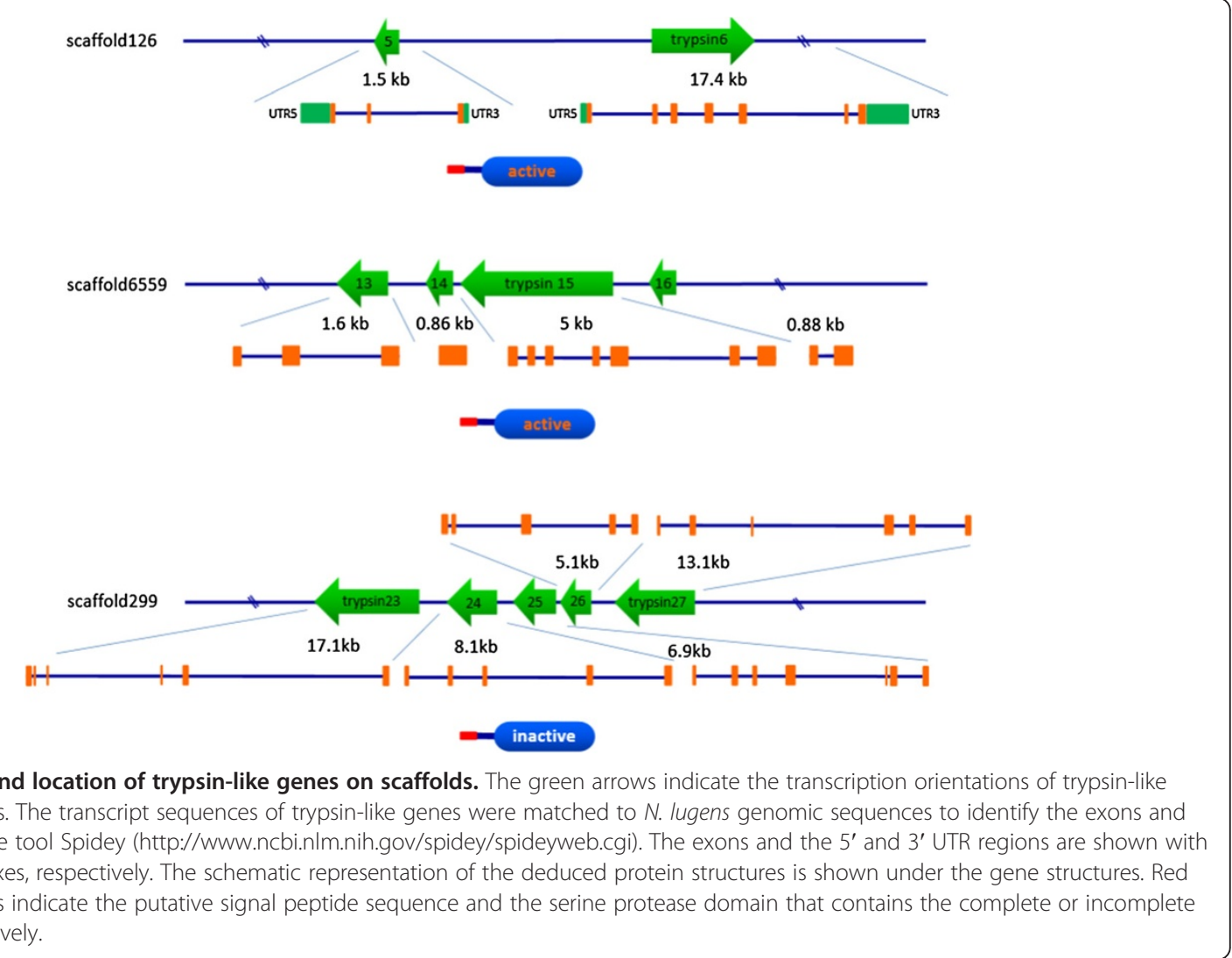

the fat body, while trypsin 4, 7, 19 and 29 genes were observed to have very high expression levels in the carcass. Similar tissue-specific expression patterns and tandem distribution at the same scaffolds indicate that some trypsin-like genes, i.e., trypsin 23-27, most likely have similar functions. However, some genes, i.e., trypsin 13-16, despite having identified sequences and locations in the $N$. lugens genome, had no available transcript sequences, suggesting that they are possible pseudogenes.

A comparison of the genome-available insect species revealed that the trypsin-like genes could have undergone a major expansion in Diptera (i.e., Culex quinquefasciatus 196, Aedes aegypti 184, Anopheles gambiae 181, D. melanogaster 152), Coleoptera (i.e., T. castaneum 104) and Lepidoptera (i.e., B. mori 84), but not in Hymenoptera or Hemiptera. Apis mellifera has few trypsin-like genes (40), which could reflect its colonial feeding strategy that alleviates the pressure on the digestion system for individual insects. Among all genome-sequenced insects, two plant phloem sap-feeding Hemiptera insects, $N$. lugens (31) and Acyrthosiphon pisum (34), possess the least number of trypsin-like genes, accounting for only about one-fifth of that in Diptera insects. $N$. lugens and $A$. pisum have evolved to survive on a nutritionally imbalanced diet of phloem sap, i.e., simple sugars and amino acids. This imbalanced diet is compensated by the intracellular symbionts, which provide essential nutritional components that are absent in phloem. It is likely that the abundant digestion enzymes are not necessary when these insects utilize the phloem sap as their nutrition source. Therefore, it might be a reasonable strategy to reduce the number of digestive proteases in these insect species when compared to the necessity of abundant trypsin-like serine proteases in leaf-feeding silkworm, the grain-feeding red flour beetle and the polyphagous dipteran insects.

\section{Immune-related serine protease genes}

Serine proteases play very important roles in the innate immune responses of invertebrate animals. These proteases are mainly involved in the processes of melanin formation and hemolymph coagulation against the infections of foreign pathogens. Melanin formation is mediated by the prophenoloxidase (proPO) activating cascade, which has been extensively studied in many insect species [18-21]. Several serine proteases and serine protease homologues, i.e., prophenoloxidase activating enzymes with clip domains, are known as the major humoral immune factors in the regulation of the melanin reaction in the proPO cascade $[20,22]$. The hemolymphclotting phenomenon was first identified as a prominent 


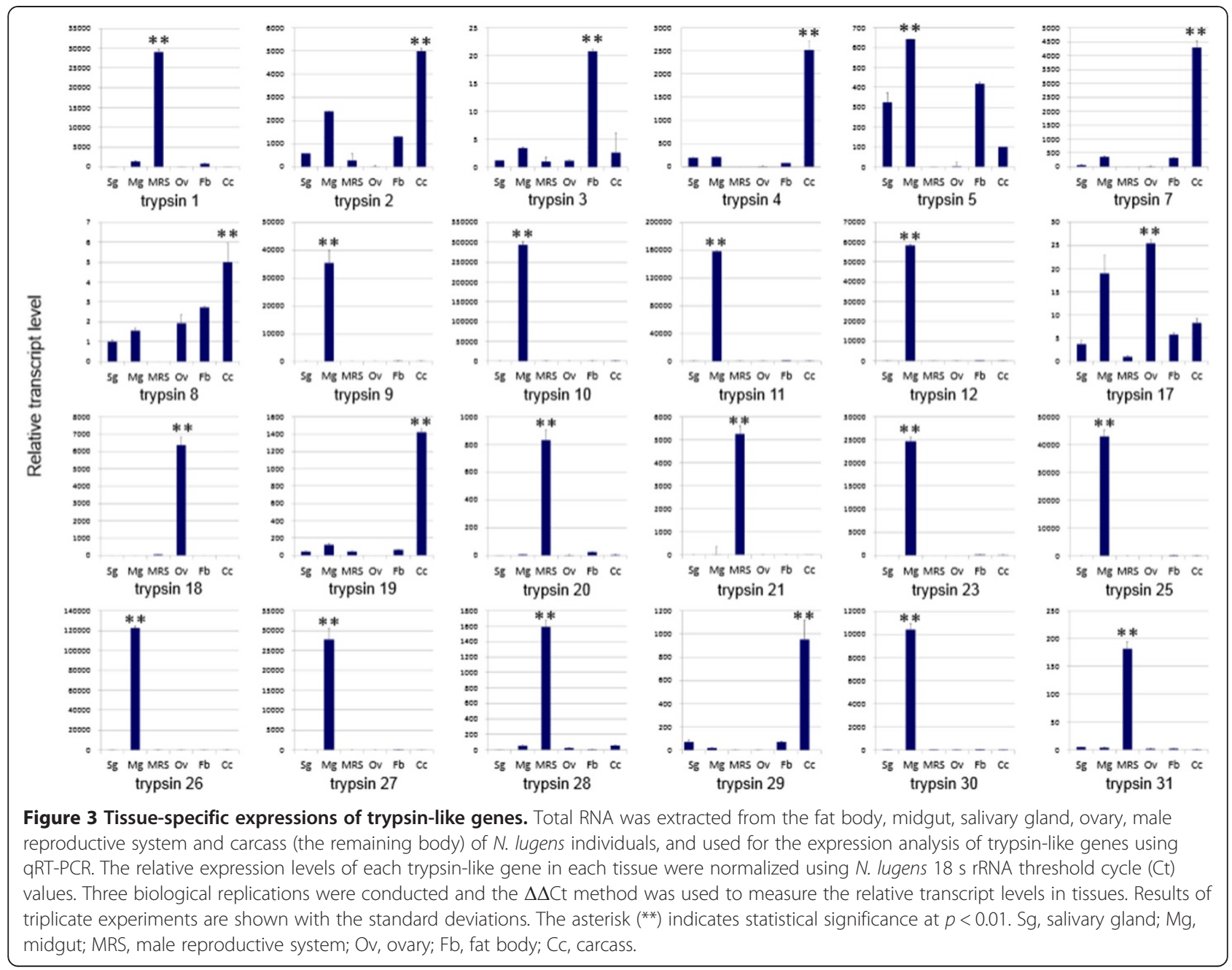

defense system in the horseshoe crab (Limulus polyphemus). Bacteria-triggered hemolymph clotting was mediated via the coagulation cascade, which consists of three principle serine protease zymogens, namely clotting factor $\mathrm{C}$, clotting factor $\mathrm{B}$ and a proclotting enzyme. Clotting factor $\mathrm{C}$ acts as a biosensor to respond to lipopolysaccharide (LPS), a major cell wall component of gram-negative bacteria, and to activate clotting factor $\mathrm{B}$, which in turn converts the proclotting enzyme to a clotting enzyme, which leads to hemolymph clotting. In contrast to non-insect arthropods, little is known about the molecular basis of hemolymph coagulation in insects.

In this study, we identified a limulus clotting factor $\mathrm{C}$ like gene in the $N$. lugens genome. This gene features two complement control protein (CCP) domains. The CCP module, also known as the SUSHI domain, contains approximately 60 amino acid residues and has been identified in complement factors in the mammalian blood coagulation system. The $N$. lugens clotting factor $\mathrm{C}$ like gene consists of a signal peptide, three consecutive low-density lipoprotein receptor class A domains (LDLa), two CCP domains and a serine protease domain with the catalytic triad (Figure 4A). We found that this domain structure seems to be insect-specific, as it has been not observed in vertebrates and noninsect arthropods yet. A comparison of the homologous genes from insect species revealed that some clotting factor C like genes contain only one CCP domain, which is found in A. pisum and the lepidopteran insects Bombyx mori, Maduca sexta and Danaus plexippus. In contrast, the genes from hymenopteran insects, e.g., bees and ants, dipteran insects, e.g., mosquitos, and coleopteran insects, e.g., red flour beetles, include two CCP domains (Figure 4A). Phylogenetic analysis shows that the clotting factor $\mathrm{C}$ like genes of hymenopteran, dipteran and lepidopteran insects form three major clusters (Figure 4B). The N. lugens and A. pisum genes locate to an independent cluster and are closely related to each other, suggesting that they have the closest phylogenetic relationship among the compared insect species. Despite the unclear functions, the domain compositions of these genes could be helpful in 


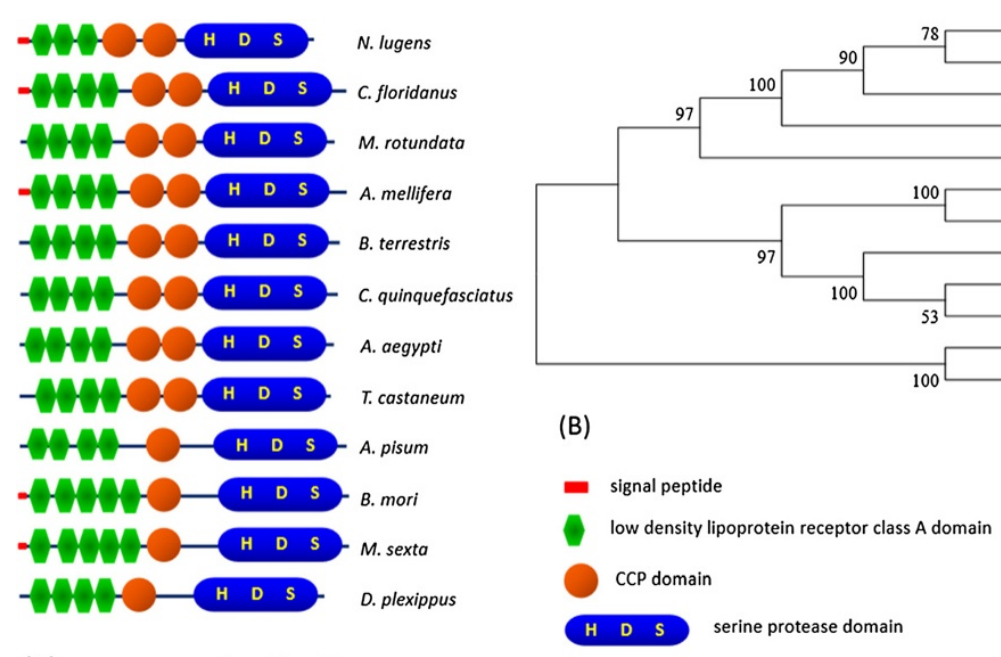

(A)

Figure 4 Analysis of clotting factor C like genes in insects. (A) Schematic representation of clotting factor C-like proteins from insect species Red bars, green hexagons, orange circle and blue oblongs indicate the putative signal peptide sequence, LDLa domain, CCP domain and serine protease domain with the catalytic triad, respectively. The size bar indicates the amino acid residues of the deduced proteins. (B) Phylogenetic analysis of insect clotting factor $C$ like genes. The phylogenetic tree was constructed based on the deduced amino acid sequences of clotting factor $C$ like genes by maximum likelihood, using the program Mega 5.05 (http://www.megasoftware.net/). The Jones-Taylor-Thornton (JTT) model for amino acid substitution was used, while a test of phylogeny was carried out using the bootstrap analysis with 1000 replications. The GenBank accession numbers for the sequences are as follows: Nilaparvata lugens (KJ512060); Camponotus floridanus (EFN71201); Acyrthosiphon pisum (XP_001944706); Apis mellifera (XP_001120594); Bombus terrestris (XP_003403297); Danaus plexippus (EHJ70705); Bombyx mori (AFK93534); Culex quinquefasciatus (XP_001864236); Tribolium castaneum (XP_967486); Aedes aegypti (XP_001655952); Manduca sexta (AAR29602); Megachile rotundata (XP_003707203).

understanding the potential functions of clotting factor $\mathrm{C}$ like genes in insects, e.g., the N-terminal LDLa repeats are lipoprotein binding domains, implying the capability of carrying lipoprotein. Lipophorin, the insect equivalent of vertebrate lipid carriers, has been identified as the clotting protein in several insect species. The CCP domain and the $\mathrm{C}$-terminal serine protease domain of the $N$. lugens clotting factor $\mathrm{C}$ like gene suggest that it is a potentially active enzyme and may have the ability to recognize or bind microbe antigens. A clotting factor B like gene that showed the highest sequence similarity with a homolog of Bombus impatiens was found in the N. lugens genome. The predicted protein seems not to have catalytic activity due to the absence of an Asp residue in the deduced serine protease domain. Three proclotting enzyme genes with the characteristic clip domain were identified and reported in our recent work [13]. In this study, to understand whether the clotting factor-like genes have the immune related functions, we analyzed the bacteria-induced expressions aiming at these genes. Our results revealed that the gene expression of proclotting enzyme 1 was notably induced by both E. coli $k 12$ and B. subtilis challenge at $6 \mathrm{~h}$ p.i., before it gradually decreased to $24 \mathrm{~h}$ p.i. (Figure 5). In contrast, the expressions of proclotting enzyme 2 and proclotting enzyme 3 were barely increased by E. coli k12 and B. subtilis during 6-24 h p.i. Clotting factor $\mathrm{B}$ and $\mathrm{C}$ expressions were not activated by bacteria infection (data not shown). These results indicate that proclotting enzyme 1 quickly responded to the invasion of foreign bacteria and may have a role in the host defense response. Despite the functions of the putative $N$. lugens clotting factors are not understood, the identification of these candidate genes makes it worthwhile to carry out further functional analyses because they provide us with a more comprehensive grasp and a better understanding of insect immune mechanisms.

\section{Other serine protease genes}

Insects depend on extracellular serine protease cascades to achieve their various physiological processes.

Nudel, gastrulation defective (Gd), snake and easter constitute the major components of the extracellular signal cascade in the Toll-Dorsal pathway. In insects, the best-characterized function of the Toll-Dorsal pathway is the establishment of the dorsal-ventral axis in early embryonic patterning. This pathway also contributes to other processes at later developmental stages, such as immune response, morphogenetic movements and muscle development [23]. A search of the $N$. lugens genome revealed a series of serine protease genes that include one nudel like, one gastrulation defective (Gd), 12 snakes and 15 easters (Table 1 ). In addition, five stubble-like genes were identified from the $N$. lugens genome. 


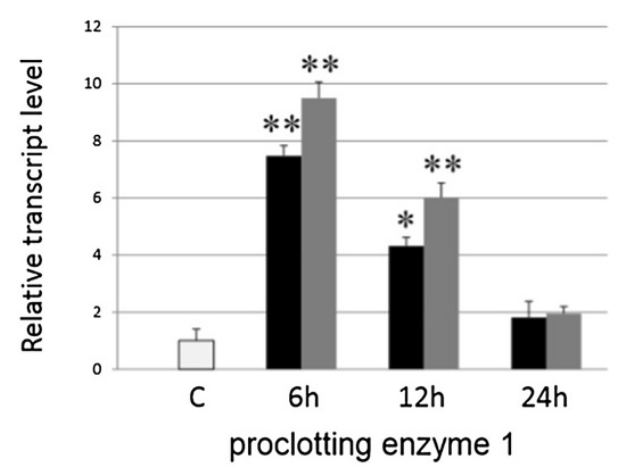

$$
\begin{aligned}
& \text { E.colik12 } \\
& \text { B.subtilis } \\
& \text { } P B S
\end{aligned}
$$
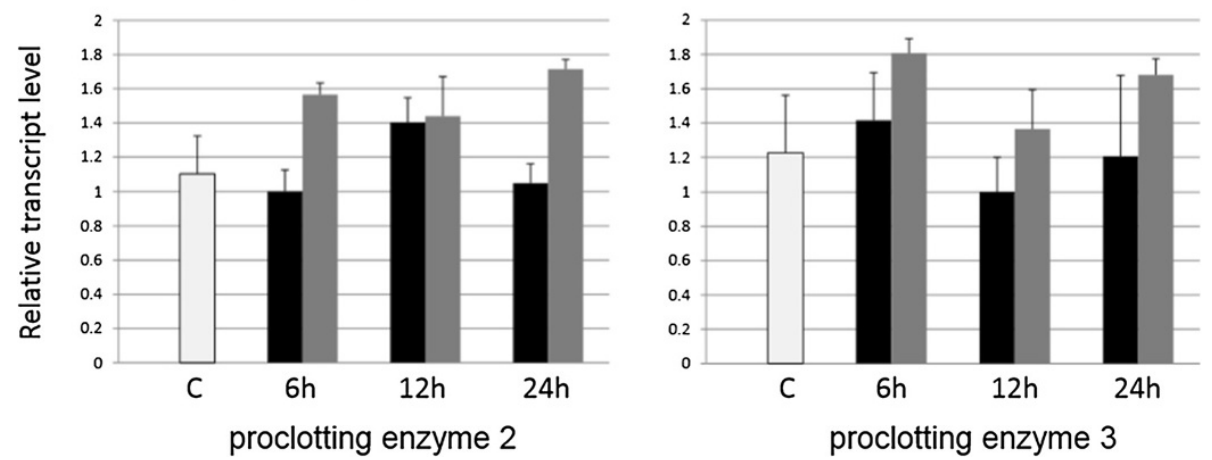

Figure 5 Bacteria-induced gene expressions of the putative clotting factors. Fifth instar nymphs were microinjected with $E$. coli $\mathrm{K} 12$ or B. subtilis. Total RNA was extracted from the nymphs at the indicated times after injection. PBS-injected sample was used as a control. The relative expression levels of each gene at different time points were normalized using the $N$. lugens $18 \mathrm{~s}$ rRNA Ct values. Three biological replications were conducted and the relative transcript levels at each time point were calculated using the $\Delta \Delta \mathrm{Ct}$ method. Results of triplicate experiments are shown with the standard deviations. The asterisk $\left(^{* *}\right)$ indicates statistical significance at $p<0.01$ between the PBS-injected and bacteria-injected nymphs. *indicates statistical significance at $p<0.05$ between the PBS-injected and bacteria-injected nymphs. The E. coli K12 and B. subtilis injected samples are shown on the left (black) and right (dark gray), respectively. C refers to the PBS-injected control. 6,12 and $24 \mathrm{~h}$ refer to bacteria-injected specimens at 6, 12 and $24 \mathrm{~h}$ p.i.

The $N$. lugens nudel like gene, which has an identical domain structure and significant sequence similarity to the $A$. pisum nudel like gene, consists of a signal peptide, four consecutive LDLa repeats at its $\mathrm{N}$-terminus and a serine protease domain at the $\mathrm{C}$-terminus (Figure 6A). The phylogenetic tree indicates that the $N$. lugens and $A$. pisum genes are closely related to each other and form an independent cluster, but are distantly located from the homologs of other insect species, i.e., bees, ants and mosquitoes, which contain a transmembrane region and 8-10 LDLa repeats and thus form another independent cluster (Figure 6B). The presence of the putative signal peptide sequences in the $N$. lugens and $A$. pisum nudel like genes suggest that their protease products are secreted. The $N$. lugens $\mathrm{Gd}$ gene contains a putative signal peptide and a serine protease domain, which are phylogenetically most closely related to counterparts from several bees of the hymenoptera insects (Figure 6C). Unlike the characteristic easter genes that have a clip domain in some insect species, i.e., D. melanogaster and A. mellifera, the $N$. lugens easter genes (10 SPs and 5 SPHs) only contain a signal peptide and a serine protease domain but lack the clip domain. The predicted protein products of the $N$. lugens easter gene have approximately 300-400 amino acids and show significant sequence identities with the Harpegnathos saltator Easter protease (Table 1). Most easter genes are located at the same scaffolds with the same or opposite transcription orientations, i.e., easter 1-6 genes closely locate at scaffold 258, easter 7-8 genes locate at scaffold 574 and easter 9-11 genes locate at scaffold 1012 (Figure 6D), implying that gene duplications occurred in the genome. The $N$. lugens easter genes contain multiple exons (Figure 6D and Table 1). Some easter genes, i.e., easter 5-6 and easter 9-10 most likely lost their catalytic triads during the gene duplication process, which generated the inactive proteases with unknown functions. Seven snake genes have been identified in our recent work. Like most snake genes characterized thus far, these genes possesses a clip-domain [13]. Clip-domain serine proteases play important roles in mediating innate immunity and embryonic development [24]. In this study, we confirmed the additional five snake like genes lacking a clip domain and named them snake like 8-12. Most snake like genes closely locate at the same scaffold, i.e., snake 1 and snake 5-10 locate at scaffold 407 with different 


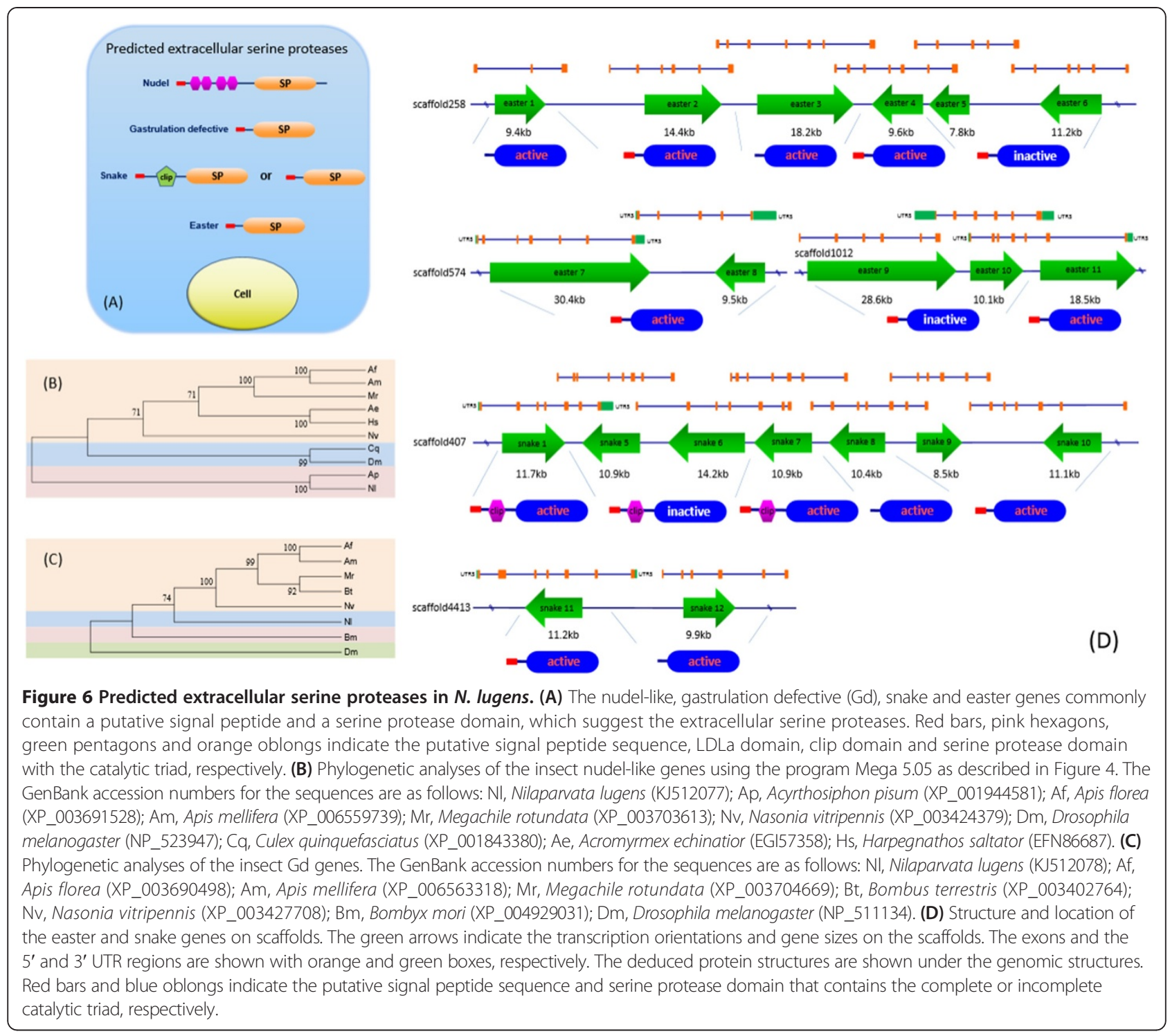

transcription orientations, while snake 11 and 12 locate at scaffold 4413 (Figure 6D). The $N$. lugens snake genes consist of 6-8 exons (Figure 6D and Table 1). The tandem distribution of two or more snake genes in a scaffold suggests that this gene family likely took place the gene expansion during the evolutionary process, which generated a group of homologues genes. The $N$. lugens nudel, Gd, snake and easter candidate genes commonly contain a signal peptide and serine protease domain, suggests they probably function in the extracellular space (Figure 6A). The in-depth elucidation of these serine proteases will be necessary to understand their potential roles in the physiological processes. Five serine protease genes showed the highest sequence similarities to the stubble genes of hymenoptera insects, i.e., bees and ants. Stubble is an integral membrane protein required for imaginal disc morphogenesis in D. melanogaster. However,
$N$. lugens stubble like genes do not contain transmembrane regions but have a predicted signal peptide, implying that they are secreted proteases (Table 1). N. lugens stubble like genes consist of 5-7 exons. Their deduced protein products range from 324 to 467 amino acids. Some stubble like genes distribute at a scaffold, i.e., stubble 1 and 2 locate at scaffold 126 (Table 1), which indicates the possible gene duplication. In this study, the identification of the variant snake, easter and stubble like genes suggests a possibility that they could contribute to the substrate specificity and provides useful insights into the physiological processes in this insect species.

\section{Development and sex-specific expression}

In our previous study, we obtained $N$. lugens development and sex-specific expression profile data from eggs, 2nd instar nymphs, 5th instar nymphs and female and 
male adults [12]. In this study, we analyzed the expressions of the entire SP and SPH genes in the different developmental stages and sexes. The genes displaying the significantly differential expressions are shown in Figure 7. Trypsin genes exhibited the various expression patterns. Trypsin 8 was solely expressed in 2 nd instar nymphs, while trypsin 2 was expressed in both 2nd and 5th instar nymphs. The transcripts of trypsin 3 and trypsin 23 genes were detected during nymph and adult stages, but were not detectable or were expressed at extremely low levels in eggs. The expressions of trypsin 4 and trypsin 6 genes significantly increased between the egg and 5th nymph stages. Trypsin 5 and trypsin 7 had significantly high transcript levels in the egg and 2nd nymph stages, suggesting that they may function in early developmental stages. Trypsin 24 transcripts were detected at the highest level in 5th instar nymphs followed by 2 nd nymphs and female adults, but were hardly detected in eggs or male adults. Trypsin 22 showed a distinct expression pattern, with maximum transcript levels detected in 2nd instar nymphs followed by eggs and no expressions in 5 th instar nymphs or female adults. Trypsin 20, trypsin 21 and trypsin 28 displayed similar expression patterns and were detected at high levels in 5th nymphs and male adults. The expression of trypsin 18 was restricted to female adults. Snake genes were thought to be involved in immune responses and embryonic development in insects. In our previous study, we reported that several snake genes, including snake 1 , snake 2 and snake 5 , were highly expressed in male adults. In this study, we found that the transcript of snake 4 gene was exclusively detected in 5 th instar nymphs. Another snake gene, snake 11 also showed the highest expression level in 5 th instar nymphs followed by 2 nd instar nymphs. The detailed functions and relationship between the snake genes needs to be further clarified. Interestingly, almost all easter genes displayed similar expression

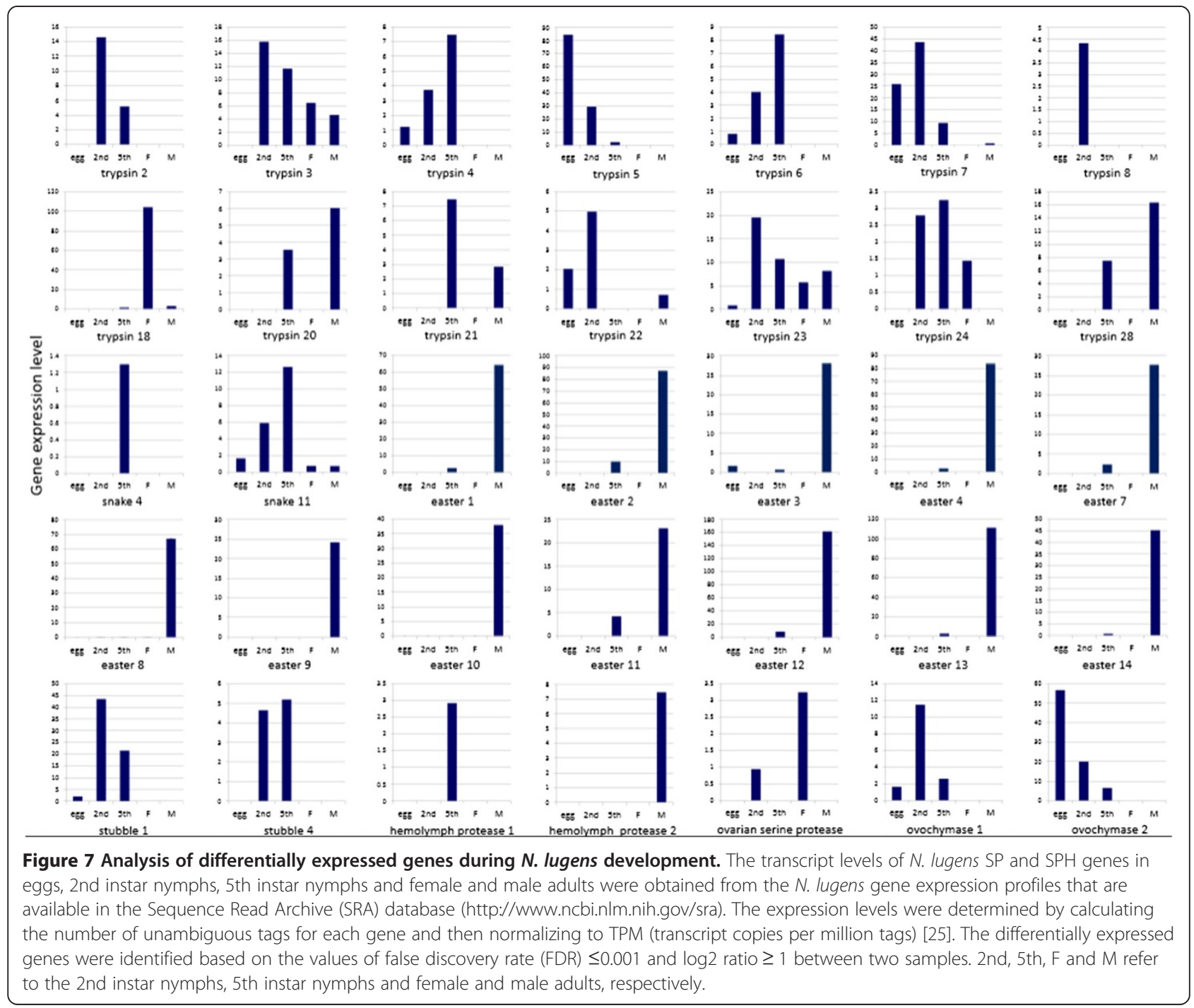


patterns, having notably high transcript levels in male adults despite having very low levels in 5th instar nymphs. These results suggest that the easter genes seem to be the male-specific expressions, which may be vital for the reproduction or development of $N$. lugens male individuals. Stubble genes, including stubble 1 and stubble 4, were mainly expressed in 2nd and 5th instar nymphs and were found at extremely low levels or barely detectable in eggs and adults. In addition, several serine protease genes displayed specific expression patterns. Of the two hemolymph protease genes, one homolog (GenBank accession no. KJ512090) was exclusively expressed in 5th instar nymphs while the other (GenBank accession no. KJ512091) was observed to be specific to male adults. A transmembrane serine protease gene (GenBank accession no. KJ512109), a homolog of the
A. pisum ovochymase 1 gene, showed much higher expression levels in 2nd instar nymphs than 5th instar nymphs and eggs. The other two transmembrane serine protease genes, (GenBank accession no. KJ512111 and KJ512110), which showed high sequence similarities to the Tribolium castaneum ovarian serine protease and ovochymase 2, respectively, were highly expressed in female adults or eggs, implying their potential reproduction-associated functions. The widely different expression patterns suggest that $N$. lugens serine proteases may have multiple functions during the developmental process. To confirm the gene expression data from high-throughput Illumina sequencing, we selected sixteen genes to analyze their developmentand sex-specific expressions using qRT-PCR. As a result, the expression patterns of these genes are coincident with the expression profile (Figure 8).

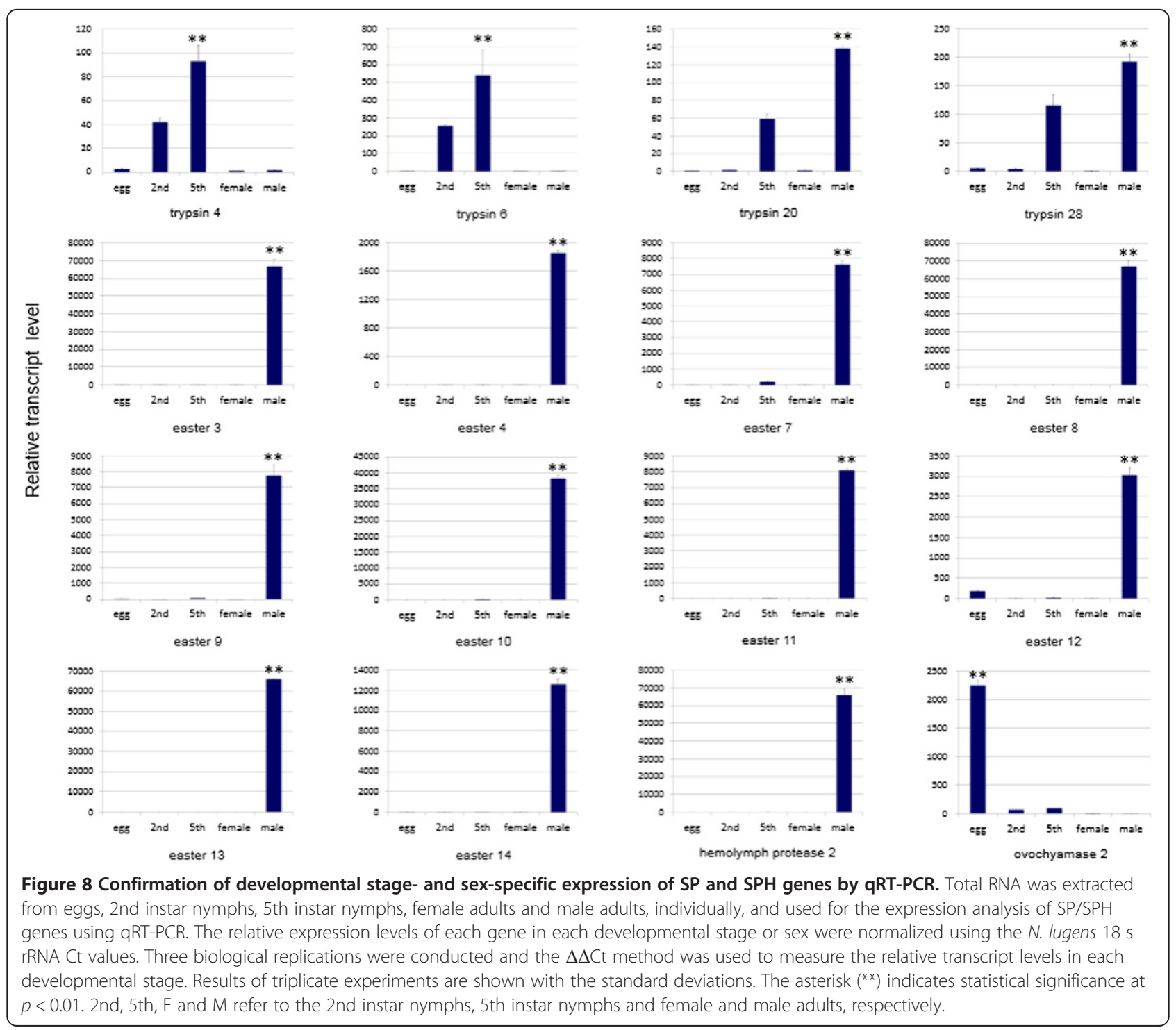




\section{Conclusions}

The accomplishment of sequencing the entire $N$. lugens genome makes it possible to fully identify a large gene family such as the serine protease family in a monophagous sap-sucking arthropod herbivore. N. lugens SP and SPH genes display different tissue-, development-, sexspecific and bacteria-induced expression patterns, which provide meaningful clues for a better understanding of the digestive, developmental, reproductive and immunological mechanisms in this insect species. It is of interest and necessary to determine their functional significance as this could be helpful in clarifying the detailed physiological mechanisms in $N$. lugens and could provide potential targets for the management of this pest in the future.

\section{Availability of supporting data}

The supporting data in this study have been submitted to the open access repositories. The $N$. lugens transcriptomic dataset and gene expression profile datasets are available in the Sequence Read Archive (SRA) database (http://www.ncbi.nlm.nih.gov/sra). The accession number of the $N$. lugens transcriptomic dataset is SRX023419. The accession numbers of the $N$. lugens gene expression profile datasets are as follows: eggs (SRX023493), 2nd instar nymphs (SRX023492), 5th instar nymphs (SRX023494), macropterous female adults (MFA) (SRX023495), macropterous male adults (MMA) (SRX023496) and brachypterous female adults (BFA) (SRX023497). The transcript sequences of $N$. lugens SP and SPH genes were submitted to National Center for Biotechnology Information (http:// www.ncbi.nlm.nih.gov/). The accession numbers of these sequences are as follows: KJ512060-KJ512142, KC355213KC355215 and KC355219-KC355225. We also provide the nucleotide and protein sequences of SPs/SPHs as an Additional file 2: List S1 in this manuscript. The phylogenetic trees of the clotting factor C-like genes (submission ID: 15882), nudel-like genes (submission ID: 15883) and gastrulation defective (Gd) genes (submission ID: 15884) were deposited in TreeBASE, a database of phylogenetic information (http://treebase.org/treebaseweb/user/submissionList.html).

\section{Additional files}

Additional file 1: Table S1. Gene-specific primers used in qRT-PCR analysis.

Additional file 2: List S1. The nucleotide and deduced protein sequences of $\mathrm{N}$. lugens SP and SPHS.

\section{Abbreviations}

SP: Serine protease; SPH: Serine protease homolog; proPO: Prophenoloxidase; CLIP: Clip-domain serine protease; CDS: Coding sequence; His: Histidine; Asp: Aspartic acid; Ser: Serine; FDR: False discovery rate;

LPS: Lipopolysaccharide; DEPC: Diethylpyrocarbonate; NTC: No-template control; qRT-PCR: Quantitative real-time PCR; CCP: Complement control protein; LDLa: Low-density lipoprotein receptor class A domain; Gd: Gastrulation defective; MRS: Male reproductive system; ML: Maximum likelihood.

\section{Competing interests}

The authors declare that they have no competing interests.

\section{Authors' contributions}

YYB conceived and designed the experiments and wrote the manuscript. YYB analyzed the N. lugens genome, transcriptome and gene expression profile data. XQ and BY performed the experiments of tissue-, development- and sex-specific gene expressions. LBC and ZCW conducted the bacteria-induced expression experiments of the immune-related serine protease genes. CXZ organized this work and provided directions. All authors discussed the results and commented on the manuscript. All authors read and approved the final manuscript.

\section{Acknowledgements}

This work was supported by National Basic Research Program of China (973 Program, No.2010CB126200) and the National Natural Science Foundation of China (Grant No. 31371934).

Received: 11 March 2014 Accepted: 17 June 2014

Published: 21 June 2014

\section{References}

1. Bass C, Carvalho R, Oliphant L, Puinean A, Field L, Nauen R, Williamson M, Moores G, Gorman K: Overexpression of a cytochrome P450 monooxygenase, CYP6ER1, is associated with resistance to imidacloprid in the brown planthopper, Nilaparvata lugens. Insect Mol Biol 2011, 20(6):763-773.

2. Bao Y-Y, Wang Y, Wu W-J, Zhao D, Xue J, Zhang B-Q, Shen Z-C, Zhang C-X: De novo intestine-specific transcriptome of the brown planthopper Nilaparvata lugens revealed potential functions in digestion, detoxification and immune response. Genomics 2012, 99(4):256-264.

3. Mao Y-B, Cai W-J, Wang J-W, Hong G-J, Tao X-Y, Wang L-J, Huang Y-P, Chen $X$-Y: Silencing a cotton bollworm P450 monooxygenase gene by plant-mediated RNAi impairs larval tolerance of gossypol. Nat Biotechnol 2007, 25(11):1307-1313.

4. Baum JA, Bogaert T, Clinton W, Heck GR, Feldmann P, Ilagan O, Johnson S, Plaetinck G, Munyikwa T, Pleau M: Control of coleopteran insect pests through RNA interference. Nat Biotechnol 2007, 25(11):1322-1326.

5. Price DR, Gatehouse JA: RNAi-mediated crop protection against insects. Trends Biotechnol 2008, 26(7):393-400.

6. Zhang H, Li HC, Miao XX: Feasibility, limitation and possible solutions of RNAi-based technology for insect pest control. Insect Sci 2013 20(1):15-30

7. Zou Z, Lopez DL, Kanost MR, Evans JD, Jiang H: Comparative analysis of serine protease-related genes in the honey bee genome: possible involvement in embryonic development and innate immunity. Insect Mol Biol 2006, 15(5):603-614.

8. Rawlings ND, Barrett AJ: Evolutionary families of peptidases. Biochem J 1993, 290:205-218.

9. $Y u$ X-Q, Jiang $H$, Wang $Y$, Kanost MR: Nonproteolytic serine proteinase homologs are involved in prophenoloxidase activation in the tobacco hornworm, Manduca sexta. Insect Biochem Mol Biol 2003, 33(2):197-208.

10. Ross J, Jiang H, Kanost MR, Wang Y: Serine proteases and their homologs in the Drosophila melanogaster genome: an initial analysis of sequence conservation and phylogenetic relationships. Gene 2003, 304:117-131.

11. Zhao P, Wang G-H, Dong Z-M, Duan J, Xu P-Z, Cheng T-C, Xiang Z-H, Xia Q-Y: Genome-wide identification and expression analysis of serine proteases and homologs in the silkworm Bombyx mori. BMC Genomics 2010, 11(1):405.

12. Xue J, Bao Y-Y, Li B-I, Cheng Y-B, Peng Z-Y, Liu H, Xu H-J, Zhu Z-R, Lou Y-G, Cheng J-A: Transcriptome analysis of the brown planthopper Nilaparvata lugens. PLoS One 2010, 5(12):e14233.

13. Bao Y-Y, Qu L-Y, Zhao D, Chen L-B, Jin H-Y, Xu L-M, Cheng J-A, Zhang C-X: The genome-and transcriptome-wide analysis of innate immunity in the brown planthopper. Nilaparvata lugens. BMC Genomics 2013, 14(1):160. 
14. Bustin SA, Benes V, Garson JA, Hellemans J, Huggett J, Kubista M, Mueller R, Nolan T, Pfaffl MW, Shipley GL: The MIQE guidelines: minimum information for publication of quantitative real-time PCR experiments. Clin Chem 2009, 55(4):611-622.

15. Bao YY, Lv ZY, Liu ZB, Xue J, Xu YP, Zhang CX: Comparative analysis of Bombyx mori nucleopolyhedrovirus responsive genes in fat body and haemocyte of B. mori resistant and susceptible strains. Insect Mol Biol 2010, 19(3):347-358.

16. Thompson JD, Gibson TJ, Plewniak F, Jeanmougin F, Higgins DG: The CLUSTAL_X windows interface: flexible strategies for multiple sequence alignment aided by quality analysis tools. Nucleic Acids Res 1997, 25(24):4876-4882.

17. Tamura K, Peterson D, Peterson N, Stecher G, Nei M, Kumar S: MEGA5: molecular evolutionary genetics analysis using maximum likelihood, evolutionary distance, and maximum parsimony methods. Mol Biol Evol 2011, 28(10):2731-2739.

18. Cerenius L, Lee BL, Söderhäll K: The proPO-system: pros and cons for its role in invertebrate immunity. Trends Immunol 2008, 29(6):263-271.

19. Söderhäll I, WU C, Novotny M, Lee BL, Söderhäll K: A novel protein acts as a negative regulator of prophenoloxidase activation and melanization in the freshwater crayfish Pacifastacus leniusculus. J Biol Chem 2009, 284(10):6301-6310.

20. Kan H, Kim C-H, Kwon H-M, Park J-W, Roh K-B, Lee H, Park B-J, Zhang R, Zhang J, Söderhäll K: Molecular control of phenoloxidase-induced melanin synthesis in an insect. J Biol Chem 2008, 283(37):25316-25323.

21. Cerenius L, Kawabata S-i, Lee BL, Nonaka M, Söderhäll K: Proteolytic cascades and their involvement in invertebrate immunity. Trends Biochem Sci 2010, 35(10):575-583.

22. An C, Ishibashi J, Ragan EJ, Jiang H, Kanost MR: Functions of Manduca sexta hemolymph proteinases HP6 and HP8 in two innate immune pathways. J Biol Chem 2009, 284(29):19716-19726.

23. Belvin MP, Anderson KV: A conserved signaling pathway: the Drosophila toll-dorsal pathway. Annu Rev Cell Dev Biol 1996, 12(1):393-416.

24. Jiang $H$, Kanost MR: The clip-domain family of serine proteinases in arthropods. Insect Biochem Mol Biol 2000, 30(2):95-105.

25. Wang X-W, Luan J-B, Li J-M, Bao Y-Y, Zhang C-X, Liu S-S: De novo characterization of a whitefly transcriptome and analysis of its gene expression during development. BMC Genomics 2010, 11(1):400.

doi:10.1186/1471-2164-15-507

Cite this article as: Bao et al:: Genomic insights into the serine protease gene family and expression profile analysis in the planthopper,

Nilaparvata lugens. BMC Genomics 2014 15:507.

\section{Submit your next manuscript to BioMed Central and take full advantage of:}

- Convenient online submission

- Thorough peer review

- No space constraints or color figure charges

- Immediate publication on acceptance

- Inclusion in PubMed, CAS, Scopus and Google Scholar

- Research which is freely available for redistribution 